\title{
Effectiveness of probiotic in preventing and treating antibiotic-associated diarrhoea and/or Clostridium difficile-associated diarrhoea in patients with spinal cord injury: a protocol of systematic review of randomised controlled trials
}

Samford Wong ${ }^{1,2,3,7^{*}}$, Ali Jamous ${ }^{1,4}$, Jean O'Driscoll ${ }^{5}$, Ravi Sekhar ${ }^{6}$, Mofid Saif ${ }^{1}$, Steve O'Driscoll ${ }^{2}$, Sarah Lewis ${ }^{7}$, Eamonn McKeown ${ }^{2}$ and Shashi P. Hirani ${ }^{2}$

\begin{abstract}
Background: Probiotics may prevent antibiotic-associated and Clostridium difficile-associated diarrhoea (AAD/ CDAD). Many spinal cord injury centre (SCIC) practitioners consider probiotics generically and may not realise that efficacy can be strain-, dose- and disease-specific. In order to confirm these effects and fully evaluate the extent of probiotic effectiveness in these patients, a systematic review and meta-analysis is indicated.

Methods: The following databases will be searched for relevant studies: Cochrane Library; Centre for Reviews and Dissemination (CRD) Database; CINAHL; PsycINFO; Embase; Medline; AMED; International Clinical Trials Registry Platform Search Portal and ISRCTN Registry and will hand search a list of conference proceedings. Any randomised controlled trials without restriction of publication status will be included with treatment of AAD/CDAD. Outcomes will include the effect of probiotic on the occurrence of AAD/CDAD and duration of diarrhoea, intensive care unit admission, hospital mortality and length of hospital stay. Two reviewers will independently screen the titles, abstracts or even full texts and extract data. Two other reviewers will assess study quality. Revman 5.1 software will be used to conduct meta-analysis and calculate the risk ratio for dichotomous data. Weighted mean difference or standard mean difference will be calculated for continuous data. The Cochrane Collaboration's tool will be used to assess the risk of bias.
\end{abstract}

Discussion: This systematic review protocol will provide information on probiotic therapy for AAD and CDAD in spinal cord injury (SCI) population. The results will be disseminated through peer-reviewed publication or conference presentation.

Systematic review registration: PROSPERO CRD42015016976

Keywords: Spinal cord injury centres, Probiotics, Clostridium difficile, Antibiotic-associated diarrhoea

\footnotetext{
* Correspondence: samford.wong@buckshealthcare.nhs.uk

${ }^{1}$ National Spinal Injuries Centre, Stoke Mandeville Hospital, Aylesbury HP21

$8 \mathrm{AL}, \mathrm{UK}$

${ }^{2}$ Centre for Health Services Research, City University, London, UK

Full list of author information is available at the end of the article
} 


\section{Background Introduction}

Data on the prevalence of diarrhoea associated with antibiotic used (AAD) and Clostridium difficile (CDAD) in spinal cord injury (SCI) patients is limited. Recent metaanalysis examining randomised, double-blinded, controlled trials of probiotics in the prevention of AAD [1-4] and CDAD [4] suggest the use of probiotic is associated with a reduction in diarrhoea compared to placebo. However, the heterogeneity of the studies (varies in study population, sample size, probiotic strains, dose and duration of probiotic and definition of diarrhoea) makes it very difficult to draw definite conclusions. Many physicians and consumers view all probiotics as being the same and therefore could apply to all populations. SCI are lifechanging events that have significant medical, physical, socio-psychological effects. After SCI, patients are required to stay in SCI centres for an extended period of time before they can re-integrate back into community. Indeed, SCI patients are more vulnerable in developing $\mathrm{AAD} / \mathrm{CDAD}$ as they tend to stay in hospitals for an extended period of time after an SCI and the use of antibiotics are common. We are not aware of any published systematic review reporting the effectiveness of probiotics in preventing or treating diarrhoea in SCI patients. It appeared logical to assess probiotics in SCI patients because these patients are particularly vulnerable to diarrhoea and its consequences for many reasons, such as the increased use of antibiotics for treating urinary tract infection due to increased catheter use [5]. Diarrhoea can delay rehabilitation, increase the risk of developing pressure ulcers/delay wound healing and reduce quality of life [5].

\section{Description of the intervention}

Probiotics are live organisms that, when administered in optimum amounts, confer a health benefit on the host [6]. They are increasingly available as capsules and dairy-based food products sold in supermarkets and health food shops. Although there are numerous commercially available probiotics, there is much debate as to what beneficial effects these provide and which specific organisms may be most effective in any specific patient group $[3,7,8]$. Microorganisms commonly used in probiotic preparations include bacteria of the genera Lactobacillus, Bifidobacterium, Escherichia, Enterococcus or Bacillus and the fungal genus Saccharomyces [8].

\section{How the intervention might work}

Probiotics that colonise the gastrointestinal tract (GI) effectively help resist gut colonisation by potentially harmful bacteria. Such probiotics often have additional properties that benefit the host $[8,9]$. Certain Lactobacillus strains can produce antimicrobial compounds, known as bacteriocins, which may inhibit pathogens such as Bacillus, Staphylococcus and Enterococcus species. A specific strain of Lactobacillus acidophilus produces a bacteriocin that has shown to inhibit strains of Listeria innocua and Listeria monocytogenes [7].

\section{Why it is important to perform this review}

Different probiotic species and strains can have substantially different effects on the host $[9,10]$. Several speciesand strain-specific factors play a role in determining what benefits, if any, a probiotic may confer. To exert a beneficial effect, a probiotic must first be able to colonise the GI tract. The initial step required for GI colonisation by probiotics is adhesion to the GI mucosa [11]. Although not fully understood, current evidence suggests that the adhesive characteristics of probiotics may be due to differences in the expression of large surface proteins and their interaction with mucus-binding proteins [11]. Probiotics have been suggested as a means of preventing adverse GI conditions such as antibioticassociated diarrhoea (AAD) and Clostridium difficile-associated diarrhoea (CDAD) $[3,7,10,12]$. However, this is not a characteristic that is shared amongst all probiotic strains [8-11], and effects may differ with different patient groups $[9,12]$. One example where the use of probiotics is particularly likely to be beneficial is in patients with SCI who not only require an extended period of stay in hospital but also have increased risk of infection due to the use of urinary catheters for long-term bladder management. If diarrhoea develops, rehabilitation will be delayed, impacting not just on the patient but also causing significant extra healthcare costs. Given the severe loss of quality of life for SCI patients, if any probiotic is effective, their low cost as well as the low incidence of adverse events [6] render probiotics an attractive intervention to prevent AAD/CDAD.

Anecdotally, it has been noted that many practitioners consider probiotics in generic terms, not recognising that there may be differences between different products. Similarly, some of the healthcare facilities stock a probiotic but will not substitute one commercial probiotic for another based on cost or availability, and without regard for any scientific evidence to support the probiotic in question.

With this in mind, it is important to recognise that there is no 'generic equivalency' between probiotic species and strains. In clinical practice, it is important for clinicians to use or recommend specific commercially available probiotics that have specifically been shown to have beneficial effects in clinical trials.

To address this issue, we plan to conduct a systematic review and meta-analysis to determine: (1) if probiotic is effective in preventing or treating diarrhoea associated with antibiotic use and Clostridium difficile infection; (2) 
what is the optimal dose, duration and frequency of probiotics in SCI patients.

\section{Methods and analysis \\ Eligibility criteria for included studies \\ Type of studies}

Randomised Controlled Trials (RCTs) in English will be included without restriction of publication type.

\section{Participants}

Participants aged 18 years and over, any race or gender with a diagnosis of spinal cord injury (according to the definition of the International Standards for neurological classification of spinal cord injury and American Spinal Injury Association (ASIA) Impairment Scale (AIS) A-D [13]) will be eligible for the systematic review and metaanalysis.

\section{Type of intervention}

Probiotic administration (all strains and dose information will be recorded) in the intervention group must be given within 5 days of antibiotic commencement. The control group should receive either placebo or routine clinical care. The reason we would like to ensure the study administered probiotics within 5 days of antibiotic commencement is due to minimising the risk of dysbiosis [14].

\section{Study end points/main outcomes}

The primary study end points include the incidence of diarrhoea associated with antibiotic use and Clostridium difficile infection. The definition of diarrhoea and occurrence of $\mathrm{AAD} / \mathrm{CDAD}$ and its follow-up period will be recorded as per identified paper.

The secondary end points include duration of diarrhoea, Intensive Care Unit (ICU) admission, hospital mortality, length of hospital stay and occurrence of adverse events.

\section{Search methods for identifying studies \\ Electronic searches}

We will systematically search Cochrane Library, Centre for Reviews and Dissemination (CRD), CINAHL, PsycINFO, Embase, Medline and AMED from inception to 27th February 2015. We will also screen the reference lists of relevant studies and reviews for additional articles. In addition, we will search the following websites for unpublished or ongoing studies: International Clinical Trial Registry Platform Search Portal (http:// www.who.int/ictrp/search/en/) and ISTCTN registry (http://www.controlled-trials.com) and review abstracts from selected scientific proceedings (Proceeding of the Nutrition Society of the Nutrition Society of the UK and Clinical Nutrition Supplement or e-ESPEN of the
European Society of Parenteral and Enteral Nutrition). We will apply a language filter in this study. Studies reported in non-English language will be excluded.

\section{Search terms/search strategy}

The keywords and Medical Subject Headings related to probiotic (lactobacillus, bifidobacter*, bifidobacillus, streptococc $*$, lactococc ${ }^{*}$, leuconostoc, pediococc ${ }^{*}$, saccharomyce, probiotic and synbiotic), diarrhoea (antibiotic associated, Clostridium difficile associated) and SCI patients (spine injury, cervical injury, spine fracture, vertebra compression, vertebra dislocation, quadriplegia, paralysis, paraplegia, tetraplegia, paraplegia, thoracic injury, lumbar injury, sacral injury) will be used alone or in combination (and with synonyms and closely related words) to retrieve relevant articles. The search strategies have been developed (see Additional file 1 for Medline/ Embase/CINAHL/AMED/PsycINFO/Cochrane/Centre for Reviews and Dissemination/International Clinical Trials Registry), and a similar search strategy will be adapted for the other databases.

\section{Data collection and analysis}

Three reviewers (SW, SH and EM) will independently examine the titles and/or abstracts and eliminate irrelevant studies. The full text of all potential eligible studies will be read and their suitability for inclusion determined according to the PICO (participant, intervention, comparison and outcomes) model. Discrepancy will be resolved by consensus or discussion with other reviewers (AJ, JO'D). Inter-rater agreement will be assessed using the kappa statistics $(K<0.01$ : no agreement; $K$ : poor agreement; $K=0.21-0.4$ : fair agreement; $K=0.41-0.6$ : moderate agreement; $K=0.61-0.8$ : good agreement; $K=$ 0.81-1: very good agreement) [15]. Details of the study selection procedure are shown in Fig. 1. Excluded studies will be listed in a table with the reasons for exclusion outlined.

\section{Data extraction}

All abstract data extracted from the retrieved trials will be reviewed independently using a predefined data extraction sheet. Any discrepancy will be managed by consensus. The following variables will be recorded for each study: the name of the first author, publication year, country of origin, type of setting (SCI centres, general hospital, community), patients' characteristics (gender, age, number, inclusion and exclusion criteria), characteristics of interventions (type of probiotics, concentration, route, dose and duration of intervention), characteristics of control methods, and outcomes (occurrence of diarrhoea, mortality, ICU admission, the length of hospital stay and adverse event data, in two groups). If necessary (unclear data, missing data and extractable data), we will 


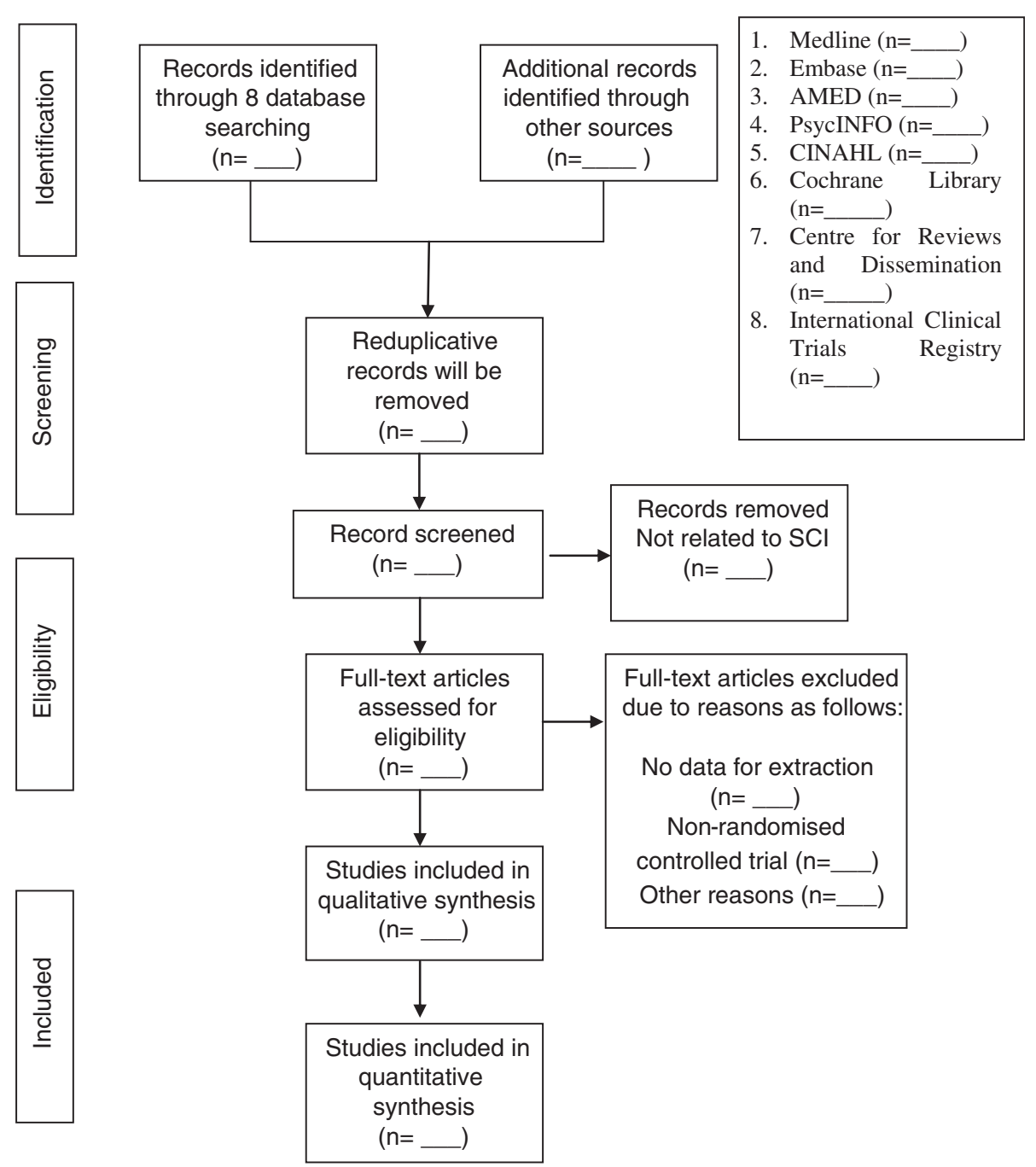

Fig. 1 Process of the systematic review

attempt to contact the corresponding authors of the included studies for missing data and for clarification.

\section{Risk of bias assessment}

The reviewers will independently assess the risk of bias using the assessment tool from the Cochrane Collaboration [16]. The following sources of bias will be detected: selection bias (random sequence generation and allocation concealment), detection bias (blinding of outcome assessment), blinding of participants and personnel (performance bias), attrition bias (incomplete outcome data), reporting bias (selective outcome reporting) and industry bias. The studies will then be classified into three levels of bias: low, unclear and high risk bias. Differences in opinion will be resolved by discussion or consultation with third reviewer (EM).

\section{Assessment of the quality of included studies}

The quality of the evidence will be rated according to the GRADE guidelines $[17,18]$.

\section{Dealing with missing data}

If there is any missing or insufficient data in included studies, we will contact the corresponding authors of the study by email or telephone to obtain more information. If we are unable to obtain the missing data, the method reported by the Cochrane Handbook for Systematic Reviews of Intervention [16] will be used to perform complete case analysis, and a sensitivity analysis will also be conducted to assess the impact of including studies with $20 \%$ or more of missing data. For all outcomes, we will conduct intention-to-treat analysis wherever possible. 


\section{Statistical analysis}

Revman (version 5.1) software will be used to conduct a meta-analysis and calculate the OR and $95 \%$ confidence interval (CI) for dichotomous data. Weighted mean difference (WMD) or standard mean difference (SMD) and $95 \%$ CI will be calculated for continuous data. If the same outcome measurement tool and unit was used, the WMD and $95 \%$ CI will be calculated, or otherwise the SMD and $95 \%$ CI. To account for differences between probiotic interventions (different strains and dose), sub-group analyses by intervention will be conducted (if we identified more than three studies using similar probiotic strains). If the intervention are too varied, data will not be pooled.

Given the heterogeneity of study designs, probiotic strains and dosing regimens, a conservative approach will be employed for all analyses based on a random effect model. The RR, WMD or SMD will be calculated by the random-effect model. Forest plot will be generated to illustrate the study-specific effect sizes along with a $95 \%$ CI.

\section{Assessment of heterogeneity}

Heterogeneity will be assessed using Cochran's $Q$ statistic and Higgins $I^{2}$ statistic, where $I^{2}>50 \%$ indicates the presence of significant heterogeneity. $I^{2}$ will be calculated according to the equation $I^{2}=100 \% \times(Q-\mathrm{df}) / Q$, where $Q$ is the Cochran heterogeneity statistic [19].

\section{Sensitivity analysis}

The sensitivity analysis will be used to assess whether the sample size and missing data impact on the results of the review. If there are adequate studies (not less than three studies), we will conduct a sensitivity analysis to check the robustness of conclusions and assess the impact of methodological quality.

For sensitivity analyses, we will perform meta-analyses using fixed effects models and assess the consistency of our results across random-effect models and fixed effects models.

\section{Assessment of publication bias}

A funnel plot will be used to evaluate publication bias if more than ten studies are included. A symmetrical funnel plot usually suggests an absence of publication bias. However, asymmetry in a funnel plot can be explained by other factors including publication bias and differing study quality [20]. We will also use Egger's test [21] to qualitatively detect publication bias.

\section{Ethics and dissemination}

Ethics approval is not required as this is a protocol for a systematic review. The findings will be disseminated in a peer-reviewed journal and presented at a relevant conference. The study is registered at PROSPERO, the
International Prospective Register of Systematic Reviews, at the University of York (CRD 42015016976).

\section{Discussion}

This systematic review and meta-analysis will use the most definitive method to assess the effectiveness of probiotic in preventing and treating diarrhoea associated with antibiotics and Clostridium difficile infection in SCI population.

The current use of probiotic to prevent and treat AAD/ CDAD remains inconclusive, where it could be limited by variation in strain, dose, duration and disease-type studied. This systematic review and meta-analysis will examine the potential impact of probiotic intervention in preventing or treating AAD/CDAD in SCI patients. Other specific aims that will be addressed by this study include determining the strains, optimal dose and duration of probiotic and the safety profile of probiotics. If probiotics are found to be effective, this may support the need for routine prescriptions to SCI patients as prophylactic or to treat AAD/ CDAD. The collaboration formed through this study will be the platform to conduct further a systematic review and meta-analysis for the probiotic management and prevention of diarrhoea in SCI patients.

\section{Additional file}

Additional file 1: The search strategies for Cochrane Library / Centre for Reviews and Dissemination database /CINAHL/ PsycINFO/ Embase/ Medline/ AMED.

\section{Abbreviations}

AAD: antibiotic-associated diarrhoea; AIS: ASIA Impairment Scale;

ASIA: American Spinal Injury Association; CDAD: Clostridium difficile-associated diarrhoea; GI: gastrointestinal tract; ICU: intensive care unit; SCI: spinal cord injury; SCIC: spinal cord injury centre.

\section{Competing interests}

SW received an unrestricted grant from Yakult Honsha for the ECLISP trial (ClinicalTrials.gov identifier: ISRCTN: 13119162). The company was aware of, but did not influence the study design and had no role in the data analysis and interpretation. The other authors declare that they have no competing interests.

Authors' contributions

SW, SH and EM contributed to the conception of the study and search strategy which was refined by SOD, SL, AJ, JOD, MS and RS. SH and EM designed the statistical analysis plan. The manuscript of the protocol was drafted by SW and was critically revised by AJ, JOD, RS, MS, EM, SH and SOD. SW registered the protocol with the PROSPERO database. EM reviewed the manuscript and will arbitrate any disagreements and ensure that no errors occur during the review. All authors have approved the publication of the protocol. This research received funding from the Research Sustainability Fund at City University London.

\section{Author details}

${ }^{1}$ National Spinal Injuries Centre, Stoke Mandeville Hospital, Aylesbury HP21 $8 \mathrm{AL}$, UK. ${ }^{2}$ Centre for Health Services Research, City University, London, UK. ${ }^{3}$ Institute for Liver and Digestive Health, University College London, London, UK. ${ }^{4}$ Royal Buckinghamshire Hospital, Aylesbury, UK. ${ }^{5}$ Department of Microbiology, Stoke Mandeville Hospital, Aylesbury, UK. ${ }^{6}$ Department of 
Gastroenterology, Stoke Mandeville Hospital, Aylesbury, UK. 'Library Services, Stoke Mandeville Hospital, Aylesbury, UK.

Received: 4 September 2015 Accepted: 16 November 2015

Published online: 24 November 2015

\section{References}

1. Cremonini F, Di Caro S, Covino M, Armuzzi A, Gabrielli M, Santarelli L, et al. Effect of different probiotic preparations on anti-helicobacter pylori therapyrelated side effects: a parallel group, triple blind, placebocontrolled study. Am J Gastroenterol. 2002; 97:2744-2749.

2. D'Souza AL, Rajkumar C, Cooke J, Bulpitt CJ. Probiotics in prevention of antibiotic associated diarrhoea:meta-analysis. BMJ. 2002;324:1361.

3. Hempel S, Newberry SJ, Maher AR, Wang Z, Miles JN, Shanman R, et al. Probiotics for the prevention and treatment of antibiotic-associated diarrhea: a systematic review and meta-analysis. JAMA. 2012;307:1959-1969.

4. Goldenberg JZ, Ma SS, Saxton JD, Martzen MR, Vandvik PO, Thorlund K, et al. Probiotics for the prevention of Clostridium difficile-associated diarrhea in adults and children. Cochrane Database Syst Rev.2013;5:CD006095. Doi: 10.1002/14651858.cd006095.pub3.

5. Consortium for Spinal Cord Medicine. Early acute management in adults with spinal cord injury: a clinical practice guideline for health-care providers. Washington DC: Paralyzed Veterans of America; 2008.

6. Food and Agricultural Organization of the United Nations and World Health Organization. Health and nutritional properties of probiotics in food including powder milk with live lactic acid bacteria. World Health Organization [online], http://who.int/foodsafety/fs_management/en/ probiotic_guidelines.pdf. 2001.

7. Todorov SC, Furtado DN, Saad SM, Gombossy de Melo Franco DB. Bacteriocin production and resistance to drugs are advantageous features for Lactobacillus acidophilus La-14, a potential probiotic strain. New Microbiol. 2011;34:357-70.

8. Ohashi Y, Ushida K. Health-beneficial effects - a review. Int J Med Microbiol. 2009;300:57-62.

9. Hill C, Guarner F, Reid G, Gibson G, Merenstein DJ, Pot B, et al. The International Scientific Association for Probiotics and Prebiotics consensus statement on the scope and appropriate use of the term probiotic. Nat Rev Gastroenterol Hepatol. 2014;11:506-14.

10. Hickson M. Probiotics in the prevention of antibiotic-associated diarrhoea and Clostridium difficile infection. Ther Adv Gastroenterol. 2011;4:185-97.

11. Tassell V, Miller M. Lactobacillus adhesion to mucus. Nutrients. 2011:3:613-36

12. Wong S, Jamous A, O'Driscoll J, Sekhar R, Weldon M, Yau CY, et al. A Lactobacillus casei Shirota probiotic drink reduces antibiotic-associated diarrhoea in patients with spinal cord injuries: a randomized controlled trial. Br J Nutr. 2014;111:672-8.

13. American Spinal Injury Association. International standards for neurological classification of spinal cord injury (revised 2000). Chicago: ASIA; 2002. http://asiaspinalinjury.org/elearning/ASIA_ISCOS_high.pdf [accessed 8th February 2015].

14. McFarland LV. Use of probiotics to correct dysbiosis of normal microbiota following disease or disruptive events: a systematic review. BMJ Open 4; e005047. Doi: 10.1136/bmjopen-2014-005047.

15. Landis JR, Koch GG. The measurement of observer agreement for categorical data. Biometrics. 1977;33:159.

16. Higgins JP, Altman DG, Gotzche PC, Juni P, Moher D, Oxman AD, et al. The Cochrane Collaboration's tool for assessing risk of bias in randomised trials. BMJ. 2011;343:d5928.

17. Guyatt GH, Oxman AD, Vist GE, Kunz R, Falck-Ytter Y, Alonso-Coello P, et al. GRADE: an emerging consensus on rating quality of evidence and strength of recommendations. BMJ. 2008;336:924-6.

18. Guyatt G, Oxman AD, Akl EA, Kunz R, Vist G, Brozek J, et al. GRADE guidelines: 1. Introduction-GRADE evidence profiles and summary of findings tables. J Clin Epidemiol. 2011;64:383-94.

19. Higgins JP, Thompson SG, Deeks JJ, Atman DG. Measuring inconsistency in meta-analyses. BMJ. 2003: 327: 5557-60.

20. Peters JL, Sutton AJ, Jones DR, Abrams KR, Rushton L. Contour-enhanced meta-analysis funnel plots help distinguish publication bias from other causes of asymmetry. J Clin Epidemiol. 2008;61:991-6.

21. Egger M, Davey Smith G, Schneider M, Minder C. Bias in meta-analysis detected by a simple, graphical test. BMJ. 1997;315:629-34.

\section{Submit your next manuscript to BioMed Central and we will help you at every step:}

- We accept pre-submission inquiries

- Our selector tool helps you to find the most relevant journal

- We provide round the clock customer support

- Convenient online submission

- Thorough peer review

- Inclusion in PubMed and all major indexing services

- Maximum visibility for your research

Submit your manuscript at www.biomedcentral.com/submit 